\title{
On the Integration of Random Access and DAMA Channels for the Return Link of Satellite Networks
}

\author{
Christian Kissling, Andrea Munari \\ DLR, Institute of Communications and Navigation, 82234 Weßling, Munich, Germany \\ \{christian.kissling, andrea.munari\}edlr.de
}

\begin{abstract}
Recent advances in Random Access (RA), improving throughput and loss rates, make the usage of such technique particularly interesting to complement Demand Assigned Multiple Access (DAMA) channels for the return link of satellite systems. In this paper, a novel approach for the integration of the two access strategies aimed at transmission delay optimization is presented. An algorithm for DAMA and RA delay estimation is presented, and its accuracy shown by simulations. Furthermore, the benefits of the proposed integration concept are demonstrated with significant gains in delay and throughput in moderate to high load operating regions.
\end{abstract}

\section{INTRODUCTION}

Satellite systems are steadily emerging as key actors in the field of broadband interactive services. The evolution of wellestablished commercial solutions as well as the definition of recent standards [1] have concurred to merge the traditional advantages of satellite communications, in terms of global and low-infrastructured coverage, with the possibility to have a broadband link from the end-user to the Gateway (GW), thus enabling a wide range of two-way schemes that range from interactive applications to voice and IP traffic.

The user-to-GW, or return link for these systems is typically set up following a Demand Assigned Multiple Access (DAMA) approach, so that ground terminals can enjoy dedicated resources to transmit their data upon allocation from a central coordinating unit. These solutions allow for high delivery reliability, yet suffer from intrinsic inefficiencies and face severe performance degradation under frequently variable or highly bursty traffic profiles. Under such conditions, in fact, static allocation of bandwidth to ground stations may not be able to effectively track the actual rate requirements, whereas dynamic policies that assign capacity only upon request incur significant delays due to the large round trip time needed for the channel set up.

In order to overcome these issues, recent solutions such as DVB-RCS2 [1] foresee the possibility to also transmit data units over shared resources that are independently used by terminals in an uncoordinated fashion following Aloha-based link layer policies. The availability of channels that can be accessed autonomously allows ground stations to promptly react to unpredicted bursts of traffic that cannot be served over the current DAMA allocation, avoiding the burden of capacity re-

Part of this work was carried out under the ESA project "Efficient networking and MAC techniques in next generation interactive broadband satellite networks", ESTEC CONTRACT No. 4000102637/11/NL/NR. quests procedures in terms of latency and additional overhead. On the other hand, resorting to uncoordinated medium access entails the risk of collisions among concurrent transmissions and of severe packet loss rates, especially under high load conditions or for large user populations. In this perspective, a large deal of research has been devoted to the improvement of delivery reliability for Random Access (RA) schemes over the return link, both in terms of congestion control algorithms and of advanced decoding techniques based on Successive Interference Cancellation (SIC) [2], [3], achieving throughput as high as $0.8 \mathrm{pk} / \mathrm{slot}$ in practical scenarios.

Despite the described benefits offered by the use of RA channels, the exclusive allocation to uncoordinated access of resources that could otherwise be reserved to additional DAMA carriers may not be overall appealing, in view of the lower efficiency that RA policies intrinsically undergo in terms of channel reuse. As a result, the integration of the two transmission techniques for delivering data traffic, while being acknowledged as a potential performance enabler [1], [4], has seen only a few algorithms [5], and has not found practical implementations to the best of the authors' knowledge.

Starting from these remarks, we introduce in this paper a novel approach capable of reaping the benefits of data uplink over random access without draining bandwidth from DAMA. To this aim, we take advantage of RA Allocation Channels (ACs) that are already present in return link satellite systems for asynchronous signalling, and opportunistically rely on them to transmit data units when a quicker delivery with respect to DAMA can be experienced. The proposed algorithm enables improvements in terms of latency and throughput, while fulfilling the twofold goal of providing a desired level of reliability and avoiding degradation for signalling performance. The outcome of this work is thus a lightweight and easily implementable solution, whose behavior is extensively investigated by means of network simulations. In addition, the design approach that we pursue brings other relevant contributions, which can be summarized as follows:

- the fundamental requirements and characteristics of an effective data relocation policy between random access and scheduled channels are identified and discussed;

- simple yet efficient delay estimation algorithms for data transmissions over both RA- and DAMA-ACs are devised. A good level of accuracy is achieved in a wide range of network and traffic configurations by properly 
capturing the key drivers for the delivery latency;

- even though the results in the present paper are obtained with reference to a DVB-RCS2-based system, the principles and algorithms that are proposed can be extended to any return link broadband satellite system.

The remainder of this paper is organized as follows. Sec. II introduces the system model, while Sec. III discusses the requirements for an effective RA-DAMA integration and describes the proposed solution, with the delay estimation presented in Sec. IV. The achievable performance are reported in Sec. V, while Sec. VI concludes the article.

\section{SYSTEM MODEL}

Throughout this paper we focus on the return link of a geostationary (GEO) broadband satellite system, and consider the DVB-RCS2 standard as a a baseline reference [1]. Ground terminals IP datagrams (L3 packets), and can be assigned dedicated resources for transmission in the form of time slots over a specific carrier, referred to as DAMA-AC. New datagrams may undergo some form of classification based on specific criteria, such as QoS and packet size, prior to being forwarded to a scheduling block and enqueued for transmission. The scheduler periodically monitors the state of the queues, generating reports that can be used for demanding resource allocation. On the other hand, an encapsulator triggers the scheduler at intervals, asking for data. Packet requests are generated based on the amount of resources available for transmission at the terminal, and result in the scheduler forwarding a proper number of datagrams, which are fragmented, encapsulated and delivered to the modem in the form of L2 packets. Within our work, we only focus on solicited DAMA resources, which a terminal can ask for by transmission of Capacity Request (CapReq) messages. For completeness, both volume- and rate-based demands are considered, and used in the presence of specific amounts of traffic to be served or when delivery latency constraints are present, respectively. ${ }^{1}$

Dedicated allocation channels are complemented by a carrier reserved to spontaneous and uncoordinated signalling transmission (e.g., logon and CapReq), shared among several terminals following the CRDSA random access-based policy [2]. In this case, messages are already generated in the form of L2 packets, and flow directly into dedicated queues at the scheduler, while allocation channels are identified by successive sets of slots referred to as RA blocks or transmission opportunities [1]. In spite of the high efficiency achieved by CRDSA through SIC at the receiver, congestion control mechanisms are needed in order to provide a sufficient level of reliability for critical, and typically not acknowledged, messages. To this aim, according to the DVB-RCS2 normative, whenever packets are present in the random access buffer of a terminal which is not already undergoing backoff or transmission procedures, a decision whether to access the medium immediately (with probability $1-p_{b}$ ) or to defer its activity for $T_{B O}$ seconds (with probability $p_{b}$ ) is made. When

\footnotetext{
${ }^{1}$ More precisely, in the DVB-RCS2 jargon, we consider in our analysis and
} simulations VBDC, AVBDC and RBDC capacity requests. the AC is eventually accessed, the terminal sends L 2 fragments deciding whether to continue at each step with probability $1-p_{b}$, and not exceeding a maximum of $N_{\max }$ L2 packets per RA block. The procedure continues until either the buffer is emptied or $L_{\max }$ consecutive RA blocks are accessed. In the latter case, the node is enforced to suspend transmissions over the AC for $I$ RA blocks prior to re-initiating the presented algorithm.

\section{INTEGRATION OF RA-AND DAMA-ACS}

The baseline architecture presented in Sec. II for the return link of a broadband satellite system resorts to dedicated resource allocation for delivering data, reserving RA-ACs to the transmission of signalling messages. While such an approach attains a high reliability, it suffers from significant performance degradation in the presence of bursty data flows. In fact, as soon as users experience sudden or unpredicted changes in the amount of traffic to serve, additional resources have to be issued, which cannot be satisfied prior than a round trip time, i.e., $\simeq 500 \mathrm{~ms}$ for the GEO system under consideration. Such an additional and penalizing delay is further magnified when the network is congested, as incoming requests may not be immediately honoured. In order to counteract this drawback, we introduce a novel solution based on opportunistic relocation of data to RA-ACs originally intended for signalling purposes. The key idea behind our approach is to let users autonomously decide whether to transmit buffered packets over dedicated or shared resources based on current availability, with the aim of minimizing delivery delay while satisfying QoS requirements for the traffic being served. The additional degree of freedom offered to terminals allows them to quickly react to unpredicted peaks of traffic, avoiding the additional latency induced by capacity allocation processes. On the other hand, the uncoordinated use of common channels poses challenges that have to be carefully addressed for the overall design to be effective, both from a user and from a system perspective. As to the former, a terminal shall clearly resort to a random access channel only when the delay experienced for delivering data over it is shorter than the one achievable using DAMA. In fact, opportunistic relocation may not always be convenient due to the restrictions imposed by congestion control policies for RA-ACs (see Sec. II), and dedicated algorithms capable of accurately comparing the expected latencies undergone using different transmission solutions have to be devised. Delay considerations, however, cannot represent the only driver for the terminal, but have to be complemented by quality constraints that specific classes of traffic may have to fulfill. In the first place, L3 QoS requirements have to be taken into account. As an example, some applications may be sensitive to out of order delivery of IP packets (e.g., TCP flows) or to high delay jitter (e.g., VoIP). Should datagrams of one such stream be served partly over DAMA and partly over RA, different transmission times could be experienced, with detrimental effects at the decoder side. On the other hand, limitations on reliability coming from the lower layers shall be also considered. From this viewpoint, the relocation of a large L3 


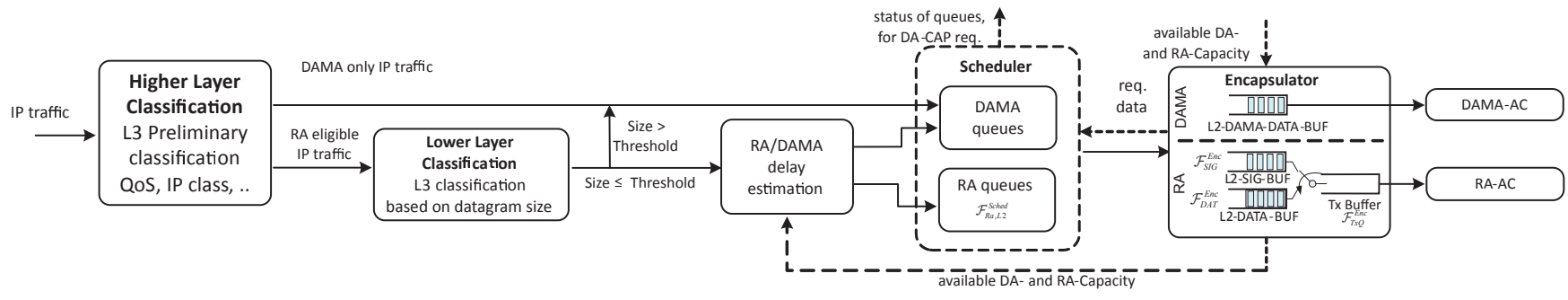

Fig. 1. Proposed RA - DAMA integration strategy

datagram to RA would result in a high number of L2 fragments to be independently sent over a channel intrinsically affected by collisions and by a lower success rate compared to DAMA. In turn, the loss of too many fragments may not be tolerable for some applications, so that a fine tuning of the delivery probability over RA is necessary not to void the benefits achievable by shorter delays for properly received packets. An effective design has then also to evaluate the possible impact of moving additional traffic to a resource which is critical from a broader perspective, as potentially quicker data deliveries shall not go to the detriment of signalling efficiency. In fact, relocation policies that overload RA-ACs or that significantly delay the transmission of capacity requests or coordination messages may end up in worsening the overal system performance. Along this line of reasoning, a properly designed prioritization mechanism among fragments that flow through signalling channels is to be devised.

Starting from these remarks, we propose the RA-DAMA integration architecture sketched in Fig. 1. Generated datagrams undergo a first classification that takes higher layer requirements into account, e.g., in terms of QoS or IP application, to identify data flows that may be split among DAMA- and RAACs. Data units that are not suitable for dynamic relocation are directly forwarded to the scheduler and enqueued following the baseline scheme presented in Sec. II. Packets eligible for random access transmission from a L3 perspective, instead, are further classified based on lower layer criteria. As discussed earlier, we consider a selection process simply based on datagram size, so that L3 data units above a certain length are enforced to go over dedicated resources and flow into the scheduler. The threshold, whose derivation is not reported here due to space constraints, can easily be determined by the GW for any target L3 packet delivery ratio, taking into account the RA-AC operating point induced by the congestion control parameters under use and the specific transmission policy in place (i.e., CRDSA). Finally, for short enough IP datagrams, the decision whether to go for an opportunistic relocation is made by comparing the delays that would be undergone for delivery over dedicated and shared resources. The latency estimation algorithm clearly represents a key point for the effectiveness of the proposed scheme, and will be described in greater details in Sec. IV. L3 packets that are eventually identified for transmission over the RA signalling carrier are enqueued in dedicated buffers within the scheduler block. At the encapsulator, separate L2 RA queues are kept for signalling and IP fragments, referred to as L2-SIG-BUF (denoted $\mathcal{F}_{S I G}^{E n c}$ ) and L2-DATA-BUF (denoted $\mathcal{F}_{D A T}^{E n c}$ ), respectively. Whenever DAMA resources are available, the module issues a request for the scheduler, processes received datagrams, inserts them in the DAMA data buffer and proceeds with transmission following the baseline procedures. Conversely, if RA capacity can be exploited, i.e., if the congestion control mechanisms allow access to the shared channel, the encapsulator first checks whether signalling messages are present. In such a case, they are processed first, and allocated on the carrier. Should the L2-SIG-BUF be empty and shared resources still available, the scheduler is triggered to provide datagrams from the RA queues, which are then fragmented, prepared for transmission and forwarded to the modem for the RAAC. This approach guarantees priority to signalling, avoiding critical drawbacks for capacity request procedures as discussed earlier. The proposed integration concept has low complexity, is simple to implement and additionally provides the benefits of an efficient situation aware exploitation of the already existing resources.

\section{DELAY ESTIMATION}

In this section we introduce details of the algorithms used for estimating the transmission delay of RA and DAMA.

\section{A. Delay estimation for transmissions over RA-AC}

Since the load control parameters are known to the terminal, they can be used to compute the average RA serving rate for a L3 packet. With the notation of Sec. II, and letting $T_{\text {block }}$ be the duration of a RA block, the maximum number of L2 fragments which can be sent on average per transmission opportunity is $\mu=\left(N_{\max } \cdot L_{\max }\right) /\left[\left(L_{\max }+I\right) \cdot T_{\text {block }}\right]$.

Simplifying the load control by assuming no idle time, i.e., $I=0$, the average serving rate, considering the backoff probability $p_{b}$, can be computed as:

$$
\widehat{R}_{b}=\left(1-p_{b}\right) \cdot \mu \text {. }
$$

It should be noted that this equation holds only in the $I=0$ case, since otherwise the imminent serving rate depends on the history of previous frames, namely the number of consecutively used frames. As explained in Sec. III, signalling messages are always given priority over IP datagrams. For this reason, the delay estimation needs to distinguish L3 data packets and signalling messages. If a signalling L2 unit is generated at time $t$, only other signalling messages in front of it or currently in the transmission buffer $\mathcal{F}_{T x Q}^{E n c}$ can delay its 
processing. The total number of such messages, including the new one is then:

$$
\mathcal{F}_{R A}(t)=\mathcal{F}_{S I G}^{E n c}(t)+\mathcal{F}_{T x Q}^{E n c}(t)+1 .
$$

If a L3 packet of length $\mathcal{L}_{p}$ bytes arrives at the delay estimation module at time $t$, instead, not only all $\mathcal{F}_{S I G}^{E n c}(t)$ and $\mathcal{F}_{T x Q}^{E n c}(t)$ fragments, but also all $\mathcal{F}_{D A T}^{E n c}$ fragments waiting in the L2 DATA-BUF and all $\mathcal{F}_{R a, L 2}^{S c h e d}$ fragments belonging to L3 packets waiting in the scheduler queues have to be considered. The overall number of fragments to be sent prior to complete transmission of the new datagram over the RA-AC is then:

$$
\mathcal{F}_{R A}(t)=\mathcal{F}_{S I G}^{E n c}(t)+\mathcal{F}_{D A T}^{E n c}(t)+\mathcal{F}_{R a, L 2}^{S c h e d}(t)+\left\lceil\frac{\mathcal{L}_{p}}{\mathcal{L}_{F}^{R A}}\right\rceil,
$$

where the RA burst payload is $\mathcal{L}_{F}^{R A}$ in bytes. By means of (1)-(3), the expected delay for transmission over the RA can finally be computed as:

$$
\delta^{R A}(t)=\frac{\mathcal{F}_{R A}(t)}{\widehat{R}_{b}}
$$

\section{B. Delay estimation for transmission over DAMA}

For estimating the DAMA delay, a terminal has information on the current queue status, the sent CapReqs (type and size) and the propagation delay $T_{p}$. If the network is in moderate load conditions, i.e. the GW is able to assign the requested capacity to all terminals, the following approach can be used.

Let $t_{C R}[i]$ be the end of the Superframe (SF) in which CapReq $i$ is generated [1]. Then, the time at which the assignment for CapReq $i$ is received can be estimated considering the expected delay on the RA channel as $t_{a s s}[i]=t_{C R}[i]+$ $\delta^{R A}\left(t_{C R}[i]\right)+T_{p}$. The SF index in which the assignment of CapReq $i$ happens is thus $S F[i]=\left\lceil t_{\text {ass }}[i] / T_{F}\right\rceil$.

For the computation of the amount of capacity in $\mathrm{SF} j$, we need to consider that the maximum assignment is bounded by the capacity of one carrier, since in practical systems terminals can transmit only in one carrier at a time. With this, the expected assigned bandwidth $B[j]$ in SF $j$ gets:

$$
B[j]=\min \left\{B_{S F} ; \sum_{i \mid S F[i]=j} C R[i]\right\},
$$

where $C R[i]$ represents the amount of bytes requested in CapReq $i$ and $B_{S F}$ the maximum capacity which a terminal can be assigned in a SF. For requests where $B_{S F}$ is exceeded, the remaining capacity is considered as an extra request which gets activated in the following SF. With reference to the DVB-RCS2 standard, such cases can either happen if a large VBDC or AVBDC request was stated or if the cumulative requests from different categories, e.g., VBDC and RBDC in sum exceed the SF capacity. With (5), the cumulative assigned capacity in SF $l$ can then be computed to $B_{\text {cum }}[l]=\sum_{j=S F[i]}^{l} B[j]$. Finally, the expected delay for a packet, arriving in SF $k$ and having size $\mathcal{L}_{p}$ bytes, can then be estimated to:

$$
\delta^{D A M A}[k]=\left\{\sum_{l=k}^{\infty} \mathcal{I}_{\left\{B_{\text {cum }}[l]<\left(\mathcal{L}+\mathcal{L}_{p}\right)\right\}}\right\} \cdot T_{F},
$$

whereas $\mathcal{I}$ is the Indicator function and $\mathcal{L}$ the number of bytes waiting in front of the arriving packet.

The presented approach offers quite accurate results in low to moderate load conditions (see Sec. V), which are the most relevant for practical systems. At higher loads, the estimate will naturally get less precise, since the GW will not be able to fully or timely serve the terminal requests.

\section{NuMERICAL RESUlts}

The simulated scenario consists of a set of $N_{u}=30$ user terminals generating L3 packets according to a Poisson-Pareto distribution with arrival rate $\lambda=20 \mathrm{pkt} / \mathrm{s}$, minimum packet size $\mathcal{L}_{\text {min }}=30$ bytes, Pareto tail distribution factor 1.1 and maximum L3 packet size cutoff $\mathcal{L}_{\max }=10$ kbytes. Since we focus on the integration aspect, all generated traffic is considered as RA eligible from the higher layers point of view, i.e., only the L2 classification based on size is simulated.

The SF duration is set to $T_{F}=100 \mathrm{~ms}$. The DAMA channel is instantiated by a carrier with 97 slots, and a highly efficient waveform with $16-\mathrm{QAM}$ rate $3 / 4$ has been selected, so to create a challenging situation for the gain achieved by the proposed reallocation policy, obtaining $\mathcal{L}_{F}^{D A M A}=175$ bytes [1]. For the RA channel, instead, a robust waveform with QPSK and rate $1 / 3$ has been selected, obtaining $\mathcal{L}_{F}^{R A}=38$ bytes. The RA-AC is operated with CRDSA-3 replicas and 200 slots per RA block. The load is controlled by varying $N_{\max }$ and, to isolate the effects coming from the integration and from the load control all other parameters have been kept constant $\left(p_{b}=0, I=0, T_{B O}=0\right)$. The relocation size threshold was selected so that only L3 packets with less than 3 fragments may be eligible to go over RA.

Simulations were carried out for the proposed RA-DAMA integration scheme with delay-estimation based RA assignment (RaDa, Delay-Est-Alloc) and, for comparison, for a Random Access-DAMA (RaDa) integrated scenario where the assignment is done randomly with a $50 \%$ chance to assign an eligible packet to the RA channel (RaDa, RandomAlloc). Additionally, two DAMA-only scenarios have been simulated. The first one (DAMA-Only, Real) implements the full Radio Resource Management (RRM) mechanism in the $\mathrm{GW}$, including request scaling in overload and bin packing (see also [6]), queue measurements and CapReq generation at terminals, transmission of requests over the channel with propagation delay $T_{p}$, allocation of resources at the GW RRM, and signalling of the capacity assignments to terminals. The second DAMA only scenario (DAMA-Only, Ideal) idealizes the CapReq generation in the terminal and eliminates the propagation delay for transmission to the GW by providing the RRM immediate knowledge of the terminals queue status. This way, possible inefficiencies in the generation of CapReqs and due to the propagation delay are eliminated, obtaining an upper bound for practical systems.

The first set of simulations investigates the delay and Packet Loss Rates (PLR) dependency on the load control parameter $N_{\max }$, which determines the maximum number of unique payloads which can be sent by a terminal in a RA block. Fig. 2 shows the obtained L3 packet delays for the four schemes. The results show first of all that the proposed delay-based allocation scheme achieves the overall lowest delay and even outperforms the ideal DAMA-only scenario. As expected, an 


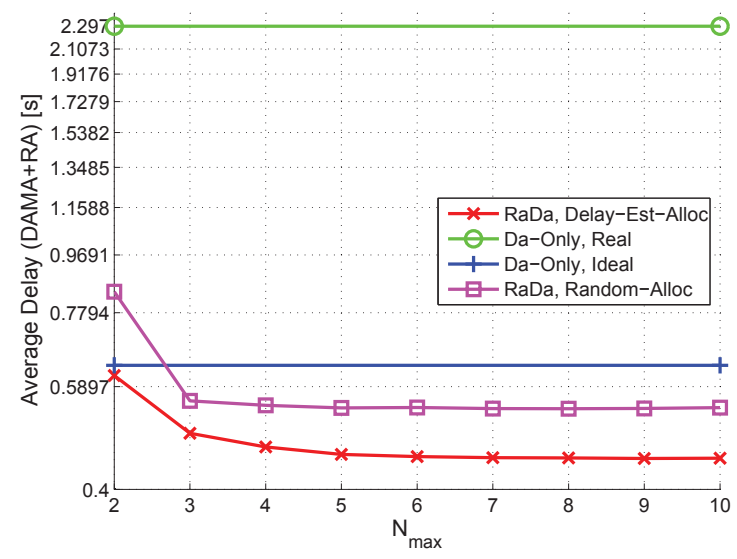

Fig. 2. L3 packet delay in dependency of load control parameter $N_{\max }$.

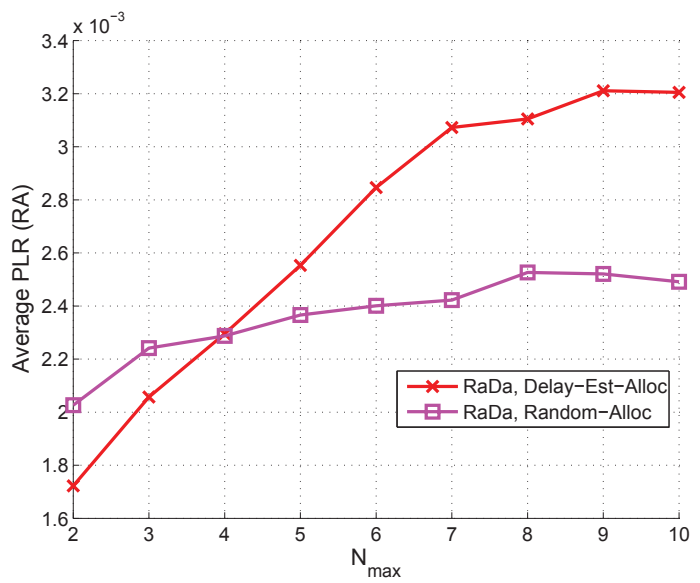

Fig. 3. L3 packet loss rate in dependency of load control parameters $N_{\max }$

increase of $N_{\max }$ reduces the delay further. In the area of low $N_{\text {max }}$, the delay gain is less significant, since the available RA capacity is mainly spent for CapReqs and only few L3 packets can be transmitted. It can be furthermore seen that DAMA-Only, real has a significantly higher delay than the ideal configuration. This is on one hand due to inefficiencies in the estimation of the future required rates and volumes, and on the other hand to the additional propagation delay.

Fig. 3 shows the impact on the L3 PLR. Since the DAMA channel is assumed error free, packets can only get lost if sent using RA. For this reason, only the two RaDa integrated scenarios are shown here. As can be seen, the delay-based allocation achieves a lower PLR than the random assignment for low settings of $N_{\max }$, while for higher values of $N_{\max }$, i.e., a more aggressive usage of the RA channel, the PLR of the delay-based allocation gets higher. The explanation for this is that for low $N_{\max }$ the delay-based allocation assigns fewer packets to the RA channel and in favor of DAMA, while the random allocation always relocates $50 \%$ of the eligible datagrams. Due to the higher load, the PLR for the random assignment gets higher. It should be noted that the random allocation has a higher delay at the same time. This is due to the stringent setting of $N_{\max }$. For a situation-agnostic $50 \%$ allocation, the RA queue is longer than for the delay-based

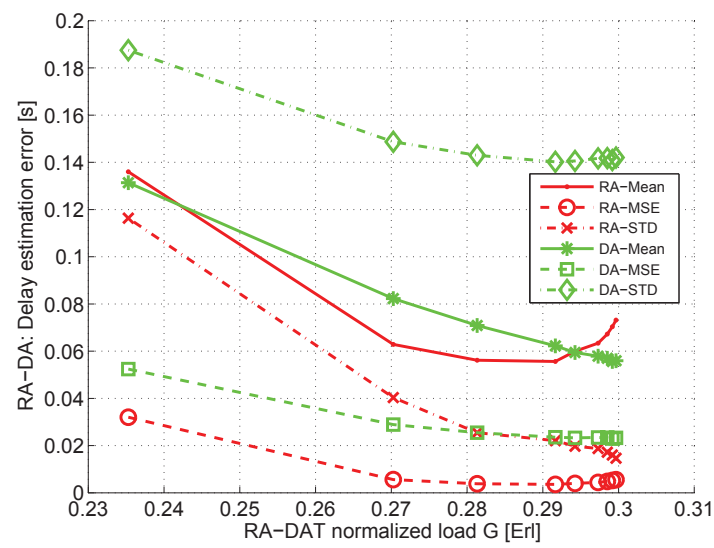

Fig. 4. Delay estimation errors for DAMA and RA.

assignment, resulting not only in a higher PLR but also in a higher delay. For $N_{\max }>4$, the PLR of the random allocation gets lower, since the delay-based assignment exploits the capacity on the RA more aggressively, resulting in a lower delay at the cost of a slightly higher PLR. Considering that the absolute PLR is anyways in the order of $10^{-3}$, the increase in PLR is anyhow not bothersome.

Fig. 4 illustrates the errors of the RA and DAMA delay estimation algorithm for L3 data packets. Besides the mean error, also the Mean Square Error (MSE) and the standard deviation are shown. First, it can be noticed that the estimation error is very small $\left(\approx T_{F}\right)$ and decreases for increasing $N_{\text {max }}$. This is because the delays are estimated at L3 packet generation time and rely on the queue conditions at this moment. Since the RA channel prioritizes signalling messages, a L3 packet waiting in the queue may have to wait longer than expected if in the meantime other signalling messages are generated. This effect gets especially evident for small $N_{\max }$, where the available RA capacity is used mainly for signalling. For higher $N_{\max }$, the bandwidth stealing of the signalling messages gets less relevant, since more data can be transmitted per SF and the estimation error decreases again. Finally, an interesting effect can be seen for $N_{\max }>5$, where the RA mean estimation error increases slightly again. The reason for this is that the RA delay estimation is based on an average rate, which is increasing jointly with $N_{\max }$. The estimation algorithm, however, does not consider that at least the end of the SF has to be awaited before the RA block can be decoded. If, due to a higher rate, the estimated arrival time is before the end of the frame while the real decoding is the end of the frame, the estimation error increases.

In order to complement our study, we have analyzed the system for a fixed value of $N_{\max }$, varying the number of terminals $N_{u}$. Fig. 5 shows a comparison of the achievable throughput of all schemes in dependence of the number of terminals present in the system, normalized to the nominal throughput of the DAMA channel. As the plot shows, all schemes perform identical up to a user population of 30 terminals. At this point, the DAMA only schemes get into saturation since all DAMA slots are used. The RaDa inte- 


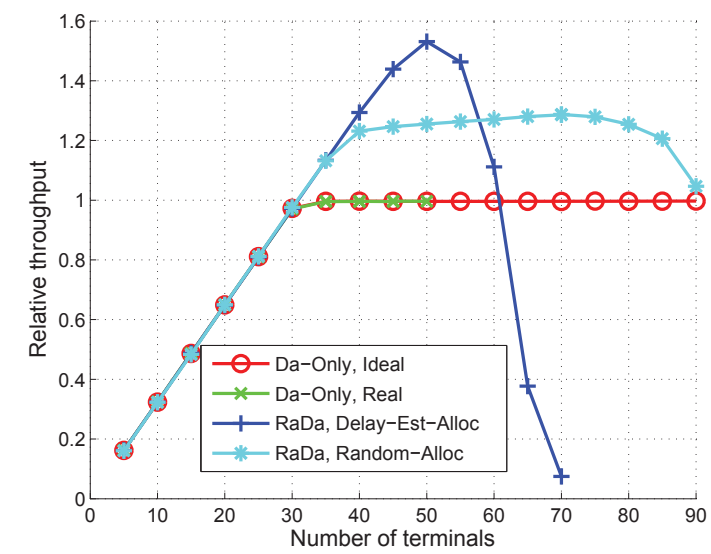

Fig. 5. Throughput normalized to max. DAMA capacity in dependency of user population $\left(N_{\max }=8\right)$.

grated schemes, however, increase further in the achievable throughput up to 1.5 times the capacity of the DAMA channel. This stems from the fact that, in comparison to the DAMAonly schemes, the additional capacity provided by the RA channel can now be also used for data transmission. For the delay-based assignment it can be seen that, after reaching a peak value, the throughput decreases rapidly, falling below DAMA-only and approaching zero. The reason for this is that, starting from 50 terminals, the load on the RA gets so high that the PLR increases drastically and not only L3 data packets but even worse also the CapReq signalling messages are lost. Without signalling, also the DAMA channel cannot work efficiently anymore and the terminals starve. A practical means in this situation would be to adjust the load control parameters, e.g., $N_{\max }$, to smaller values to lower the load.

Finally, an interesting behaviour of the random-assignment can be observed in Fig. 5. For $40 \leq N_{u} \leq 58$ terminals, the random-assignment is underperforming, which is obvious since it agnostically assigns $50 \%$ of the RA eligible traffic to the DAMA channel, even though the RA could deal with it. This also explains why the throughput gain over DAMA-only is only half of the gain of the delay-based assignment. For $N_{u}>58$, the throughput of the random assignment remains however high, even outperforming the DAMA-only system. In this region, the delay based assignment reacts on the overload in the DAMA channel by assigning packets to the RA channel, which is also already in overload (due to too high $N_{\max }$ for this region) resulting in loss of signaling and data packets. The random assignment, however, keeps the RA load lower due to the probabilistic allocation and can thus send data packets and signalling even until roughly $N_{u} \approx 75$ where also for the random assignment the overload in the RA channel gets evident, resulting in a decrease of throughput.

With respect to delay, Fig. 6 shows the results for the different integration schemes. As can be seen here, the proposed delay-based assignment achieves the lowest delay up to $\approx 58$ terminals. While the delays reached in this region are already not practicable in a real system anymore, they anyway offer an interesting insight into the different schemes. The highest

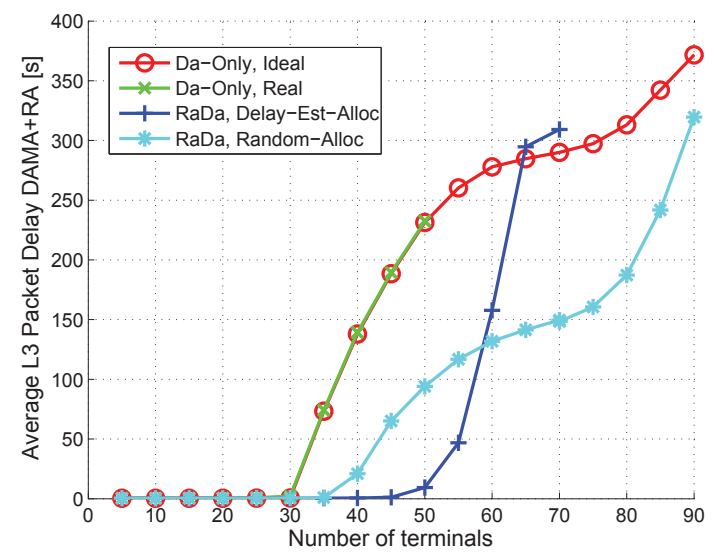

Fig. 6. Delay in dependency of user population $\left(N_{\max }=8\right)$.

delay is observed here for the DAMA only schemes (ideal and real). The random-assignment already allows a significant improvement in delay, but has anyways a much higher delay than the delay-based integration. This is again due to the fact that the random assignment does not fully exploit the capacity of the RA channel. The drastic increase in delay for the delay-based integration in the region of $N_{u}>50$ is due to the previously described overload generation in the RA channel, resulting in loss of data and signalling messages. Due to very scarce DAMA allocations in this situation, the delay grows very fast. At this point, the load control effect of the random assignment again pays off, with the delay increasing less severely for $60 \leq N_{u} \leq 80$ and outperforming the DAMA anyway. Eventually, for $N_{u} \geq 80$ the delay increases rapidly as the RA gets entirely overloaded.

\section{CONCLUSIONS}

This paper presented an approach for integrating RA- and DAMA-ACs for the return link of satellite systems by delay estimation-based packet assignment. The key design issues were discussed, and the performance evaluated by extensive simulations. The benefits and accuracy of the proposed scheme were shown, proving significant delay and throughput gains.

\section{REFERENCES}

[1] ETSI, "TS 101 545-1, Second Generation DVB Interactive Satellite System (DVB-RCS2) Part 1: Overview and System Level specification,' Tech. Rep. V1.1.1, May 2012.

[2] E. Casini, R. De Gaudenzi, and O. Herrero, "Contention Resolution Diversity Slotted ALOHA (CRDSA): An Enhanced Random Access Scheme for Satellite Access Packet Networks," Wireless Communications, IEEE Transactions on, vol. 6, no. 4, pp. 1408 -1419, April 2007.

[3] G. Liva, "Graph-Based Analysis and Optimization of Contention Resolution Diversity Slotted ALOHA," Communications, IEEE Transactions on, vol. 59, no. 2, pp. $477-487$, february 2011.

[4] R. De Gaudenzi and O. del Rio Herrero, "Advances in Random Access protocols for satellite networks," in Satellite and Space Communications, 2009. IWSSC 2009. International Workshop on, 2009, pp. $331-336$.

[5] D. Connors, B. Ryu, G. J. Pottie, and S. Dao, "A Medium Access Control Protocol for Real Time Video over High Latency Satellite Channels," Mobile Networks and Applications, 2002. [Online]. Available: http://dx.doi.org/10.1023/A:1013297627486

[6] C. Kissling, "Efficient Resource Management for a GEO MF-TDMA Return Link with Channel Adaptation," in International Communications Satellite Systems Conference (ICSSC), San Diego, CA, USA, June 2006. 